\title{
Estrategias metodológicas para la enseñanza del uso y manejo de las reglas ortográficas en el Instituto Rubén Darío de Santa Rita, Costa Caribe Norte de Nicaragua
}

\author{
Samara Sugey Sequeira Huetes 1 \\ Erlinda Mejía Martínez ${ }^{2}$ \\ Iván Jarquín Chavarría ${ }^{3}$
}

\section{Resumen}

$\mathrm{C}^{\mathrm{n}}$ esta investigación se han implementado estrategias metodológicas para la enseñanza del uso y Emanejo de las reglas ortográficas en el Instituto Público "Rubén Darío", de Santa Rita, Costa Caribe Norte de Nicaragua. Se trata de una investigación cualitativa sustentada en diseño de investigación acción participativa donde participaron estudiantes de undécimo grado, padres y madres de familia y el profesorado. Los principales instrumentos para la recopilación de la información son la entrevista, una guía de observación y un test cognitivo. Los principales resultados muestran que el estudiantado presentaba dificultades de aprendizaje en el uso y manejo de las reglas ortográficas; estas dificultades de aprendizaje han sido superadas en un 90 por ciento posteriormente a la aplicación de estrategias metodológicas. Se concluye, que el uso y manejo de las reglas ortográficas, es significativo, en el proceso de formación del estudiantado, porque su práctica, contribuye a mejorar la comunicación y escritura.

Palabras clave: reglas ortográficas; estrategias metodológicas; enseñanza; pruebas diagnósticas; aprendizaje

\section{Abstract}

In this research, methodological strategies have been implemented to teach the use and management of orthographic rules at the Public Institute Ruben Dario, Santa Rita, Nicaragua's North Caribbean Coast. This is a qualitative research based on participatory action research design involving eleventh grade students, parents and teachers. The main instruments for the collection of information are the interview, an observation guide and a cognitive test. The main results show that students had learning difficulties in the use and management of orthographic rules; These learning difficulties have been overcome by $90 \%$ after the application of methodological strategies. It is concluded that the use and management of orthographic rules is significant in the student training process because its practice contributes to improve oral and writing communication.

Keywords: Orthographic rules; methodological strategies; teaching; diagnostic tests; learning.

\footnotetext{
1 Licenciada en Ciencias de la Educación con Mención en Español. Profesora del Instituto Público, Rubén Darío. Correo: samarasequeira07@gmail.com

2 Licecnaida en Ciencias de la Educación con Mención en Español. Profesora del Instituto Público, Rubén Darío. Correo: eldinmegiamartinez@gmail.com

3 Máster en Docencia Universitaria. Coordinador de Investigación de la Universidad de las Regiones Autónomas de la Costa Caribe Nicaragüense-Recinto Universitario Las Minas. Correo: ijarquinchavarria@yahoo.com
}

Recibido: 02/05/2017 - Aprobado: 03/07/2017 


\section{Introducción}

A nivel mundial, se presentan diferentes problemas en el área del lenguaje y la comunicación, muchos estudiantes tienen grandes dificultades por distintas razones; y diferentes factores que son provenientes de padres madres de familia, de los profesores, directores y de los mismos aprendientes; paralelamente en nuestro país, también presentan las mismas dificultades, en el uso y manejo de las reglas ortográficas. En América Latina se ha producido un amplio espacio de reflexión acerca del papel de la escuela en la educación de los estudiantes, con miras a procurar la formación de ciudadanos capaces de integrarse en una sociedad globalizada (Sanders, 2013, p. 13).

La educación en Nicaragua es de forma tradicional, por lo que se presentan distintos factores, que atentan contra la calidad del proceso de enseñanza. Lo que influye de forma negativa en los y las estudiantes. El Instituto Público de Santa Rita, no es la excepción de esta problemática, específicamente en el municipio de Mulukukú. Es evidente, el poco interés que se imprime frente a esta situación sobre el uso y manejo de las reglas ortográficas. Según Hernández (2004) la ortografía puede constituir un problema mientras no haya disposición de enfrentarla, requiere de la implicación de los docentes y los estudiantes y el complejo de estrategias adecuadas de aprendizaje para que se logren alcanzar de forma creativa los hábitos y habilidades en la escritura correcta de las palabras.

Durante muchas décadas el problema más sentido, por el profesorado, es la ortografía; diariamente surgen las interrogantes: ¿De qué manera se pueden resolver esta problemática sobre el uso y manejo de la ortografía?; y ¿De qué manera se pueden implementar estrategias metodológicas que conlleven a un aprendizaje efectivo de la ortografía?. En este sentido, se genera una estrategia para la enseñanza del uso y manejo de las reglas ortograficas, logrando un mayor conocimiento de las reglas generales de acentuación en el estudiantado. Beneficiando directamente a los aprendientes y contribuyendo a la buena comunicación oral y escrita.

\section{Revisión de la literatura}

La enseñanza radica en el arte de desarrollar percepciones claras, completas y correctas de los vocablos que son objeto de estudio, sin que se margine su importancia desde el punto de vista psico- lógico, por la relación que tiene, entre la palabra y el objeto que ella designa (Puntería, 2004, p. 51). Según Torrego (2007), la enseñanza de la ortografía, está mediada por la aplicación de estrategias cognitivas a través de diferentes procedimientos como la observación, el análisis, la síntesis entre otros, que contribuyen a que los estudiantes resuelvan sus dificultades ortográficas, lo cual es de vital importancia, debido a que la práctica ortográfica favorece el buen aprendizaje en las y los estudiantes.

Acevedo (2007) en su investigación indicó que la persona que educa y aplica el constructivismo, crea un escenario agradable, atractivo y retador que permite a las y los estudiantes caminar por un sendero que los lleva a construir sus propias experiencias y a derivar las estructuras de reglas ortográficas, que le posibilitan una interpretación más profunda de la realidad, y la falta de aplicación del método constructivista es quizás el principal factor que influye en las pésimas faltas ortográficas de los estudiantes.

Dentro del lenguaje escrito, la ortografía es un elemento fundamental que sirve de ayuda para facilitar y mejorar la comunicación, Una buena ortografía es la mejor tarjeta de presentación para las personas que se comunican por escrito; dentro de esta perspectiva, es importante resaltar que en el campo de la enseñanza debe orientarse, hacia una práctica eficaz de la misma, que permita al estudiante apropiarse de elementos lingüísticos, útiles para la comprensión de cualquier área del saber.

\section{Materiales y métodos}

El presente estudio se enmarca en el paradigma cualitativo porque es una actividad sistemática orientada a la comprensión en profundidad de fenómenos sociales, a la transformación de prácticas y escenarios sociales, a la toma de decisiones y también hacia el descubrimiento y desarrollo de un cuerpo organizado de conocimiento. Desde esta perspectiva, Hernández et al. (2010), explican que en el paradigma cualitativo el enfoque o diseño "es el abordaje general que utilizará el investigador en el proceso de investigación" (p. 492). En este sentido, el diseño de esta investigación se define como investigación acción participativa cuya intención es, compartir, conocimientos con el estudiantado, a la vez generar una propuesta de estrategias metodológicas, para el uso y manejo de las reglas generales de acentuación, en donde todos y todas son protagonistas de la acción. 
Resaltamos que este estudio, se realizó en el Instituto Público "Rubén Darío", de la comunidad Santa Rita, municipio de Mulukukú, Costa Caribe Norte de Nicaragua, con la participación de 8 estudiantes de undécimo grado de los cuales 5 fueron hombres y 3 fueron mujeres, teniendo en cuenta las dificultades en el uso y manejo de las reglas generales de acentuación. Además, participaron 5 madres y 3 padres de familia, la docente y el director del centro educativo.

Las principales técnicas para la recopilación de la información que se utilizarón han sido: la entrevista abierta dirigidas a estudiantes, docentes, padres y madres de familia, y director; la observación no participantes en los procesos de clases; y test cognitivos aplicados al estudiantado. Cabe mencionar que la investigación acción fue activa y participativa porque estuvo estrechamente relacionada con la implementación de estrategias de enseñanza para la apropiación de las reglas generales de acentuación con el estudiantado de undécimo grado.

\section{Resultados y discusión}

Como referencia con los resultados del diagnóstico inicial, se procedió a elaborar las estrategias metodológicas de enseñanza aprendizaje para el uso y manejo de las reglas generales de acentuación, las que posteriormente se aplicaron por un periodo de un mes, con el fin de dar respuesta a los problemas de ortografía que aún afectaban, el proceso de enseñanza de los y las estudiantes. Los resultados logrados son: satisfactorios porque se logró que un 90\%, del estudiantado aprendiera dichas reglas ortográficas.

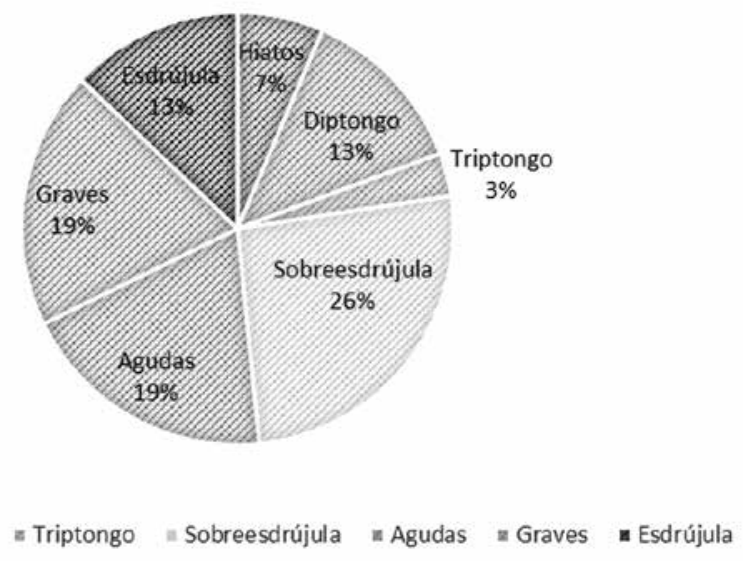

Figura 1: Diagnóstico inicial, porcentaje de estudiantes que aplican reglas generales de acentuación

Los resultados del diagnóstico inicial que se realizó a través de la lectura del poema "Ángel Guardián", de "Gabriela Mistral" a partir del cual los discentes clasificaron y subrayaron las palabras agudas, graves, esdrújulas, sobresdrújulas, hiatos, diptongos y triptongos, indicando deficiencia en el manejo y uso de las reglas generales de acentuación.

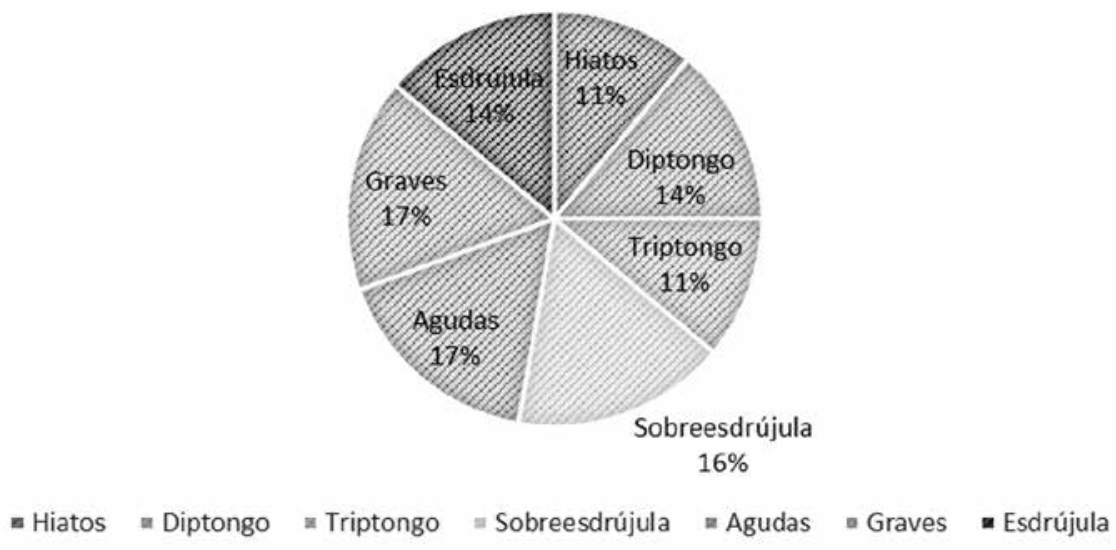

Figura 2: Diagnóstico intermedio, estudiantes que aplican reglas generales de acentuación 
Una vez aplicado el diagnóstico inicial, procedimos a aplicar el diagnóstico intermedio, el cual nos dio como resultado lo que evidencia la figura 3, del diagnóstico intermedio en donde hay un cambio, encontramos mejoras en resultados positivos.

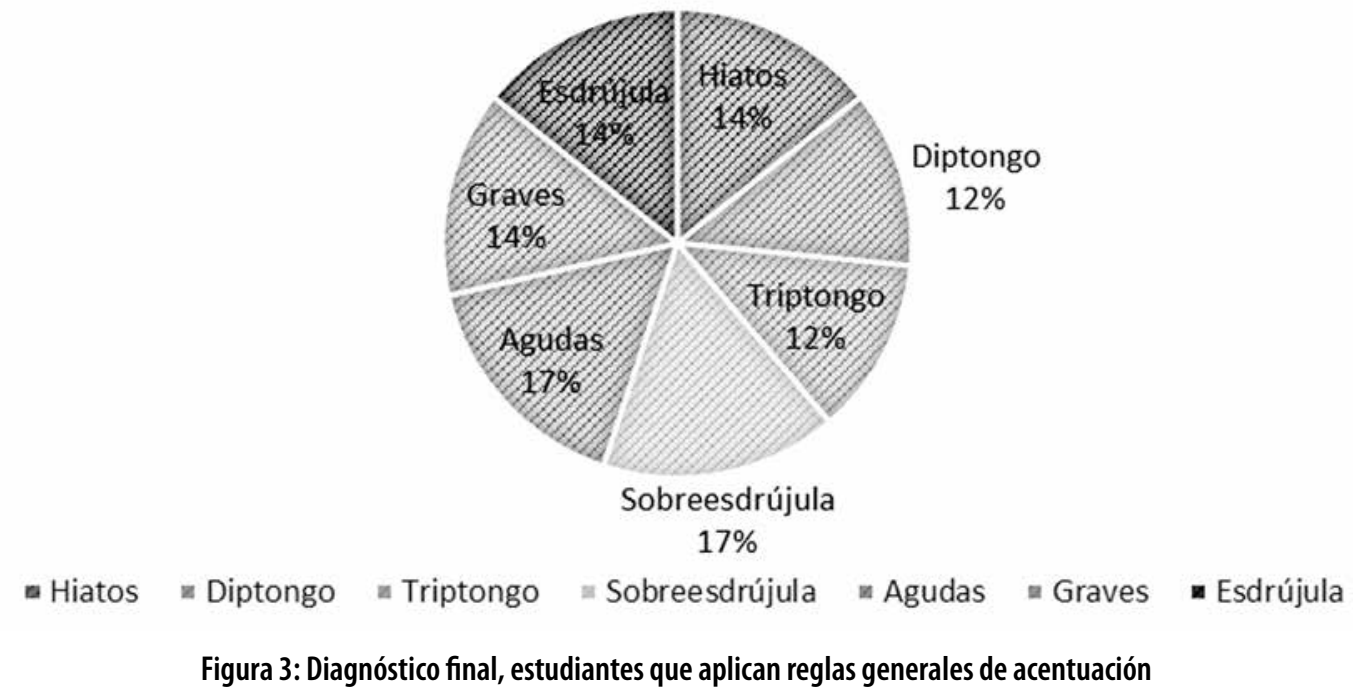

Cuando se terminó el proceso acción-participativa, se realizó el diagnóstico final, para comparar con el intermedio y el inicial, con la finalidad de corroborar si existía avances significativos en el aprendizaje del estudiantado sobre el uso y manejo de las reglas ortográficas.

Los resultados obtenidos sobre el aprendizaje del uso y manejo de las reglas ortográficas han sido satisfactorios, porque las diferentes acciones que se realizaron, como el acercamiento docente-estudiante, la implementación de estrategias metodológicas, las variadas actividades que han ayudado para evitar una clase aburrida y monótona, al mismo tiempo utilización de dinámicas integradoras y recursos de apoyos, contribuyen al aprendizaje del estudiantado.

Tomando como referencia los resultados del diagnóstico inicial se procedió a elaborar las estrategias metodológicas, de enseñanza aprendizaje para el uso y manejo de las reglas generales de acentuación, las que posteriormente han sido aplicadas. En la tabla siguiente podemos ver el porcentaje de aprendizajes que se obtuvo, en cada elemento.

Tabla 1: Aprendizajes alcanzados en los diagnósticos

\begin{tabular}{|c|c|c|c|c|c|c|}
\hline \multicolumn{7}{|c|}{ Diagnóstico inicial \% } \\
\hline Agudas & Graves & Esdrújulas & Sobresdrújula & Hiatos & Diptongos & Triptongos \\
\hline $37.5 \%$ & $37.5 \%$ & $25 \%$ & $50 \%$ & $12.5 \%$ & $25 \%$ & $6.25 \%$ \\
\hline \multicolumn{7}{|c|}{ Diagnóstico Intermedio \% } \\
\hline Agudas & Graves & Esdrújulas & Sobresdrújula & Hiatos & Diptongos & Triptongos \\
\hline $75 \%$ & $75 \%$ & $62.25 \%$ & $6.75 \%$ & $50 \%$ & $62.5 \%$ & $50 \%$ \\
\hline \multicolumn{7}{|c|}{ Diagnóstico Final \% } \\
\hline Agudas & Graves & Esdrújulas & Sobresdrújula & Hiatos & Diptongos & Triptongos \\
\hline $100 \%$ & $87.5 \%$ & $87.5 \%$ & $100 \%$ & $87.5 \%$ & $75 \%$ & $75 \%$ \\
\hline
\end{tabular}




\section{Conclusiones}

A partir de los resultados de los diagnósticos aplicados, a estudiantes de undécimo grado del Instituto público Rubén Darío de Santa Rita Mulukukú, sobre el uso y manejo de las reglas generales de acentuación, se concluye que:

- Existe la necesidad de aplicar estrategias metodológicas innovadoras para despertar el interés en el área de lectoescritura en el aula de clase, porque motiva al estudiantado.

- El estudiantado no escribe correctamente porque desconocen las reglas ortográficas para el uso de la tilde.

- Es necesario desarrollar un proceso continuo y constante en el aprendizaje ortográfico en clases.

- No se aplican adecuadamente las estrategias de enseñanza.

Desde esta perspetiva, se recomienda, la práctica de la propuesta de estrategias metodológicas que ha surgido en esta investigación acción, con el propósito de mejorar la enseñanza de las reglas ortográficas. También, aplicar estrategias que permitan al estudiantado construir su propio aprendizaje que los motive a desarrollarse, en situaciones tanto dentro como fuera del aula, para así interactuar con su medio o entorno; así mismo, promover trabajos en equipo, para una mejor interactuación del estudiantado. Algo importante es que el Ministerio de Educación (MINED), debe realizar acompañamientos pedagógicos sistemáticos y permanentes, y concluimos que el estudiantado debe autoevaluarse y cohevaluarse con el conocimiento que reconozcan el nivel de aprendizaje adquiridos y los criterios de evaluación de los aprendizajes en los que participan.

\section{Bibliografía}

Acevedo, A. S. (2007). Ortografía y gramática. Ciudad de México.

Alvarado, C. A. (2012). La ortografía. Managua: Hispamer.

Hernández, J. L. (2004). Aprendamos ortografías juntas. Managua Nicaragua

Hernández, R., Fernández, C., \& Baptista, M. (2010). Metodología de la investigación. México: McGra-Hill.

Murillo, S. H. (1996). Educación para el futuro en desarrollo. Estados Unidos

Milan, G. P ( 2014), Reglas generales de acentuación. Managua Niaragua

Punteria., J. (2004). Propuesta para aprender a puntuar. Barcelona España: Octaedro editorial.

Sanders, M. P (2013) Reglas generales de acentuación. Managua Nicaragua

Torrego, L. G. (2007). Ortografía de la Real Academia Española. Madrid: SM. 